\title{
Invited Gadolinium-Enhanced 3D Magnetic Resonance Angiography of the Thoracic Vessels
}

\author{
Mohammed A. Neimatallah, MD, ${ }^{1}$ Vincent B. Ho, MD, ${ }^{2}$ Qian Dong, MD, ${ }^{3}$ David Williams, MD, ${ }^{1}$ \\ Smita Patel, MRCP, FRCR, ${ }^{1}$ Julie H. Song, MD, ${ }^{1}$ and Martin R. Prince, MD, PhD ${ }^{3 *}$
}

MAGNETIC RESONANCE IMAGING has long been recognized as a useful tool for the non-invasive evaluation of the thoracic vasculature. Unlike computed tomography and conventional angiography, MRI is not associated with the concerns related to ionizing radiation exposure or to contrast-related nephrotoxicity. MRI is also capable of oblique image acquisition and multiplanar reformation, which aids the illustration of the thoracic vessels, inherently intertwined and complex in their arrangements. In addition, MRI using cine technique affords cardiac referenced data that enables dynamic assessment of blood flow, yielding information comparable to an echocardiogram.

Gadolinium (Gd)-enhanced three-dimensional (3D) magnetic resonance angiography (MRA) is a newer technique that provides high-resolution (ie, 3D) data very quickly and is well suited for the depiction of intrathoracic vessels. Improvements in gradient technology now allow a Gd-enhanced 3D MRA to be performed during a 20-40 second breath-hold. Because it relies on T1-shortening effects of circulating Gd-chelate contrast media and not inherent flow characteristics, Gdenhanced 3D MRA can often depict pathologic vascular segments that are not adequately visualized using unenhanced flow-based MRI techniques. In addition, Gdenhanced 3D MRA provides volumetric data that can be processed for multiplanar reformation (MPR) and maximum intensity projection (MIP) viewing. In this article, the technical considerations and potential applications for Gd-enhanced 3D MRA of the systemic and pulmonary vessels within the chest will be discussed and illustrated.

Traditionally, T1-weighted spin-echo and gradientecho pulse sequences have been employed for delineation of vascular pathology within the chest (1-10). The

${ }^{1}$ Department of Radiology, University of Michigan Medical Center, Ann Arbor, Michigan 48109-0030.

${ }^{2}$ MR Research Division, Department of Radiology, Uniformed Services University, Bethesda, Maryland 20814.

${ }^{3}$ Cornell University, New York, NY 10021 Contract Grant Sponsor: Whitaker Foundation, GE.

*Address reprint requests to: M.R.P., Cornell University, MRI Division, 416 East 55th Street, New York, NY 10021.

Received June 29, 1999; Accepted July 13, 1999.

(c) 1999 Wiley-Liss, Inc. combination of T1-weighted spin-echo and gradientecho images can often provide the information necessary for the assessment of simple clinical queries such as patency of a vessel (Fig. 1) or delineation of a vascular ring (Fig. 2). These techniques, however, rely on flowing blood (ie, the movement of blood during the acquisition period) for their illustration of vascular structures. This flow dependency makes these techniques prone to flowrelated image artifacts, thereby frequently limiting their clinical utility.

On spin-echo pulse sequences, vessels are characterized by their dark lumina. The black appearance of blood, also known as flow void, on spin-echo imaging occurs secondary to the wash-out of blood prior to the refocusing pulse and sampling of the echo. The washout may be incomplete if the echo time is too short, the vessel courses primarily within the imaging plane, or the blood flow is too slow. Incomplete wash-out results in the persistence of signal within the vessel lumen, which may result in the masking of underlying luminal pathology such as an intimal tear or the erroneous simulation of a vascular occlusion or thrombosis. A superior black blood effect is achieved by using preparatory pulses such as a double inversion pulse to null blood signal for more effective suppression even when the blood flow is slow.

Unlike spin-echo imaging, time-of-flight (TOF) and phase contrast (PC)-MRA depict flowing blood with bright signal intensity. TOF imaging relies on the wash-in or in-flow effect of unsaturated protons; PC imaging relies, on the phase shift experienced by moving protons traveling along the gradient field (7-13). Both TOF and PC imaging provide higher intra-vascular signal-tonoise ratios than spin-echo pulse sequences. These "bright blood" pulse sequences (ie, TOF and PC imaging) often show intraluminal abnormalities (Fig. 1b) not clearly identified on black blood spin-echo images. However, should blood flow be slow, turbulent, or complex, vascular signal on gradient-echo images becomes unreliable. Disturbances in blood flow can often result in signal loss, which can result in the underestimation of vessel patency, overestimation of a stenosis, or even simulation of a vascular occlusion. 

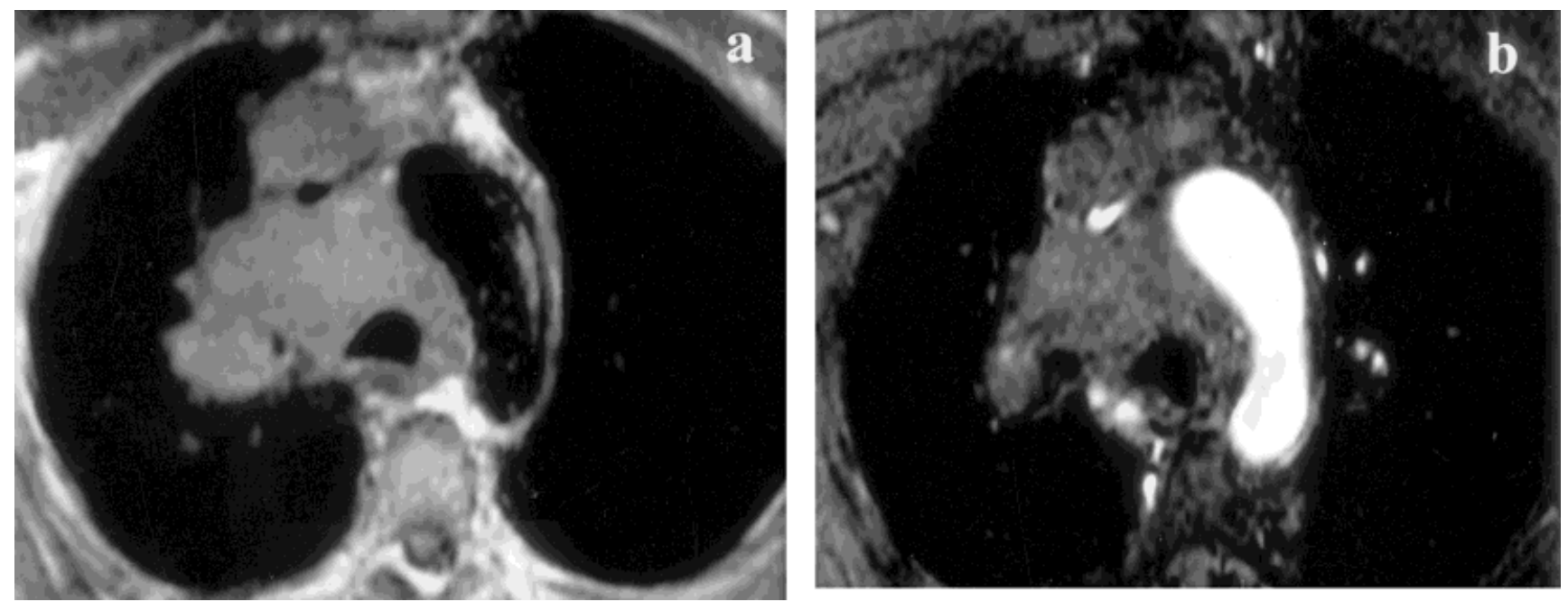

Figure 1. Axial spin echo (a) and axial 2D TOF (b) of the chest at the level of the arch showing large mediastinal mass encasing the SVC, trachea, and upper lobe branch of the pulmonary vessels. Both SE and 2D TOF depict intra- and extravascular anatomy.

Gd-enhanced 3D MRA is a relatively new technique that has the advantages of higher resolution, shorter acquisition time, speed, and improved reliability for the generation of vascular signal than the aforementioned flow-dependent MRI techniques (ie, spin-echo and gradient-echo imaging) $(3,4,6,8,9,14)$. Gd-enhanced 3D MRA often compliments traditional spin-echo and gradient-echo imaging for comprehensive evaluation of intrathoracic vascular structures.

\section{Gd-ENHANCED 3D MRA}

\section{Physical Principles of Gd-Enhanced 3D MRA}

Vascular visualization using Gd-enhanced 3D MRA relies on the $\mathrm{T} 1$ shortening of blood by Gd-chelate contrast media during its intravascular transit (13-17). This technique requires the coordination of an intravenously administered Gd-chelate contrast media bolus with image acquisition. The timing of the imaging determines the vascular territory ultimately illustrated. That

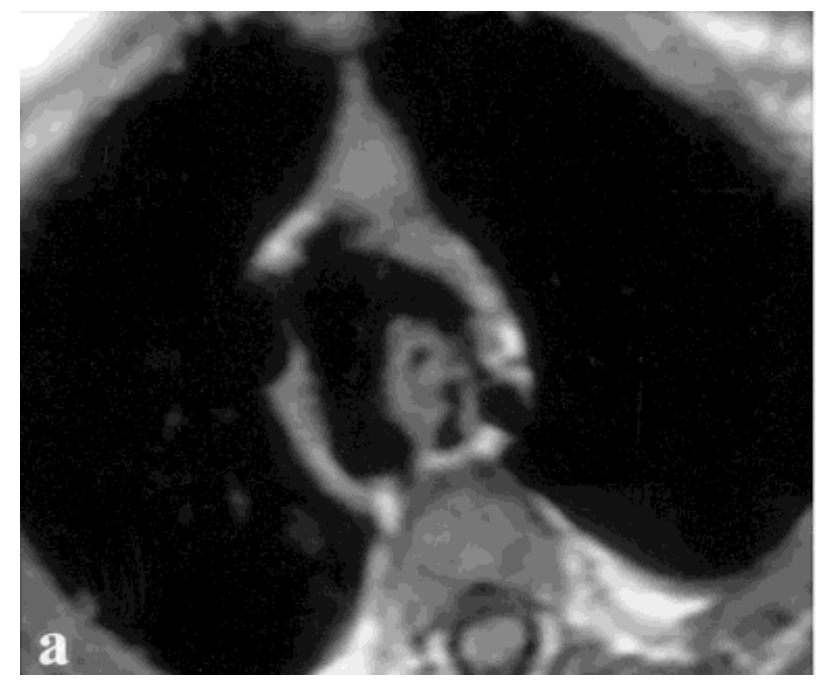

is, if the imaging is performed during the arterial phase of the contrast bolus, arteries will be preferentially illustrated. Because Gd-enhanced 3D MRA relies on T1 shortening effect of contrast media rather than on blood flow characteristics such as wash-out, TOF, or PC effects, it is less prone to flow-related artifacts and is capable of more reliably illustrating vascular structures.

Different regions of $\mathrm{k}$-space have varying importance for Gd-enhanced 3D MRA. Image contrast depends mainly on central k-space data. For most pulse sequences sequential ordering of $\mathrm{k}$-space is employed such that the center of $\mathrm{k}$-space is acquired during the middle or center of the imaging period. Since central $\mathrm{k}$-space determines image contrast, only vessels that are contrast media-enhanced during the acquisition of central k-space will be visualized on Gd-enhanced 3D MRA. Preferential arterial images can be achieved, therefore, by selectively timing the acquisition of central $\mathrm{k}$-space views during preferential arterial enhancement

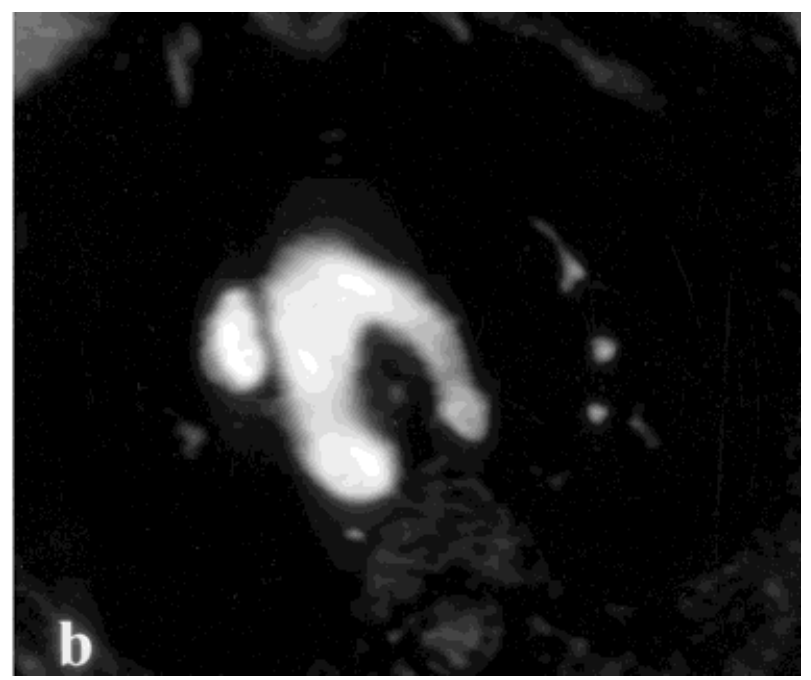

Figure 2. Double aortic arch. Axial T1 SE (a) and axial 2D TOF (b). 

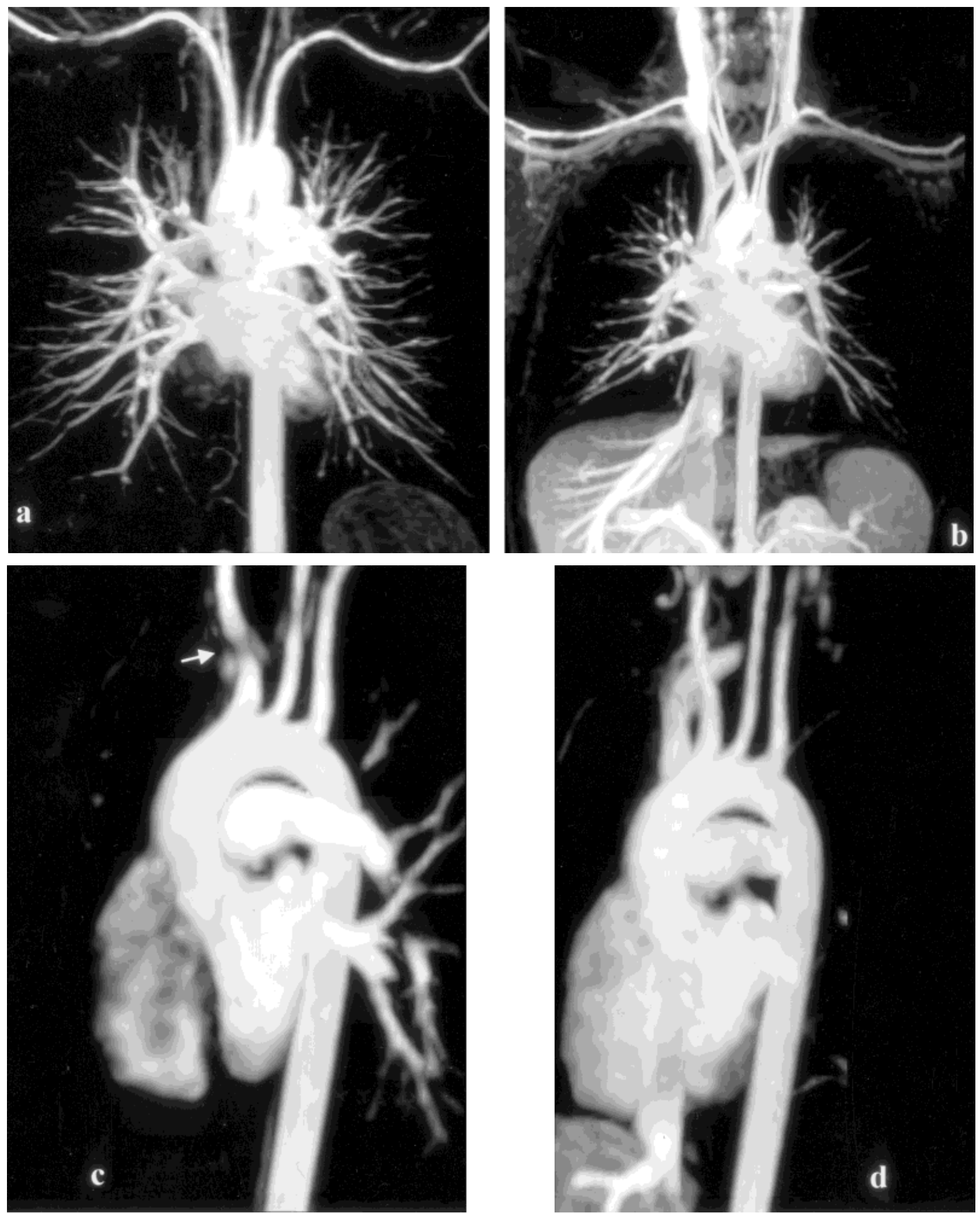

Figure 3. MIP reformats from coronal 3D Gd-MRA of normal aortic arch and the great vessels in coronal plane. Coronal reformation in (a) arterial phase and (b) equilibrium phase. Sagittal reformation in (c) arterial phase and (d) equilibrium phase. Subtle susceptibility artifact (arrow) from concentrated gadolinium during the arterial phase mimics stenosis at proximal innominate artery (c), and the venous phase confirms normal, patent innominate artery (d). 
(Fig. 3a,c). Venous structures will be seen if central $\mathrm{k}$-space lines are obtained during the venous or equilibrium phase of the contrast bolus (Fig. 3b,d). The timing of the central $\mathrm{k}$-space views relative to the contrast administration, therefore, is crucial for the resultant vascular visualization. The periphery of $\mathrm{k}$-space that contributes primarily to image resolution is also important. However, its acquisition is less time sensitive.

\section{Imaging Parameters}

For thoracic imaging, 3D Gd-enhanced MRA is performed in a breath-hold to minimize the artifacts (ie, image blurring) related to respiratory motion. For this reason, the fastest possible $3 \mathrm{D}$ imaging sequence, typically a fast 3D gradient-echo pulse (16-23), should be used. The partition dimensions (ie, partition thickness, matrix size, field of view) should be prescribed to achieve the smallest possible voxel size (eg, $2 \mathrm{~mm}$ or less in any single dimension) to afford sufficient spatial coverage of the target vessel and yet still be acquired within a breath-hold. For the thoracic aorta, the typical 3D volume employs a $2-3 \mathrm{~mm}$ partition thickness, $256 \times$ 192 matrix, and $30 \mathrm{~cm}$ field of view. On most current commercially available $1.5 \mathrm{~T}$ MR scanners, a fast 3D gradient-echo pulse sequence using the shortest possible repetition time (eg, 4-6 msec), shortest possible echo time (eg, $1-3 \mathrm{msec}$ ), and a $30-40^{\circ}$ flip angle can be performed within 20-40 seconds. The imaging time can be decreased by using partial Fourier imaging (eg, 0.5 excitation), decreased number of partitions, decreased phase encoding steps, or a rectangular field of view. Widening bandwidth also makes the scan faster but at the expense of a significant reduction in signal-to-noise ratio. These improvements in imaging speed can be used to improve image resolution (ie, decrease voxel size). Recently, image interpolation using zero-filling has gained popularity as it improves the MIP images at no additional cost to imaging time (24), although image reconstruction and post-processing time increases. It is important to note that although zero-filling generates better reformations and MIPs, it does not improve the true spatial resolution of the images.

Thoracic Gd-enhanced 3D MRA should be prescribed for a sagittal, coronal, or oblique sagittal acquisition (Fig. 4). The images should be obtained in the orientation optimal for visualization of the target structure. For illustration of the pulmonary arteries and veins, for example, Gd-enhanced 3D MRA should be performed in

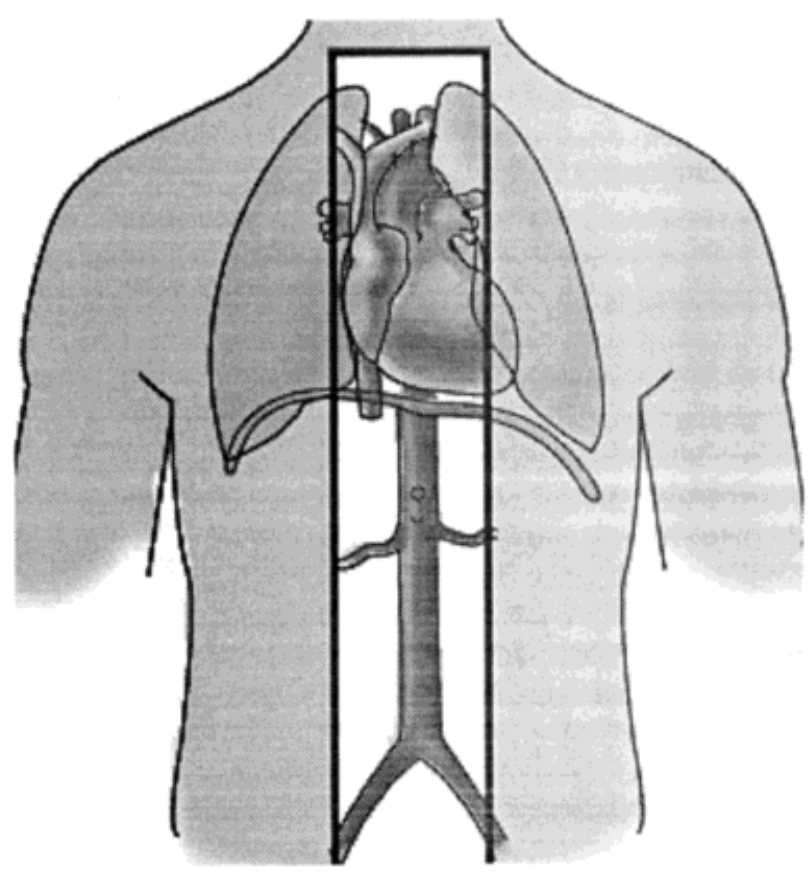

( a)

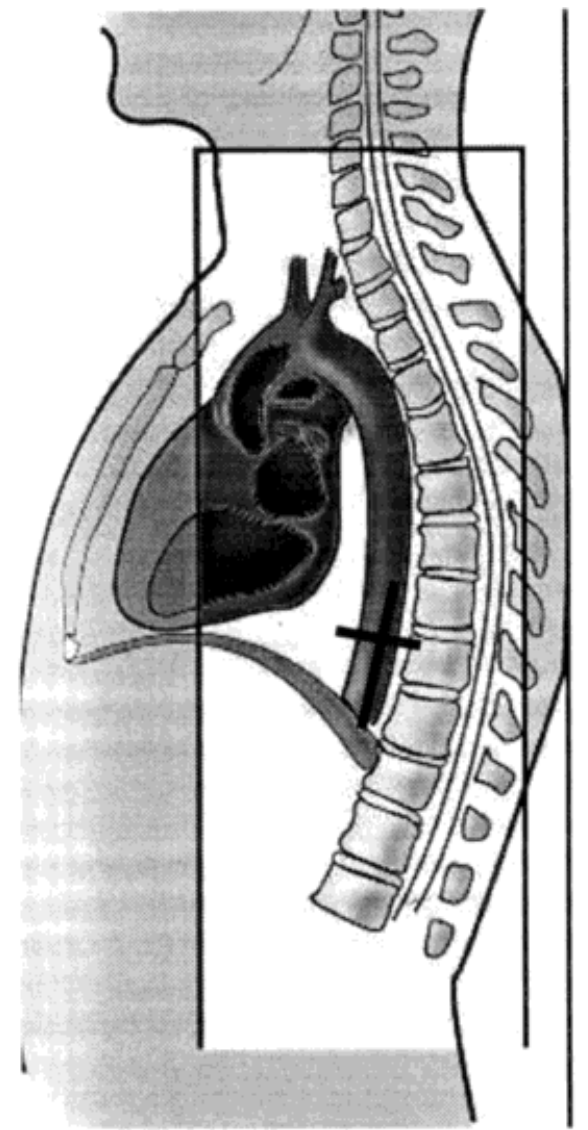

Figure 4. Illustration of coronal locator and sagittal locator demonstrating the positioning of the Volume and Tracker for coronal 3D Gd-MRA. 
the coronal plane for both lungs (Fig. 5) or sagitally for just one lung. Coronal acquisitions afford diagnostic views of the thoracic aorta and are preferable should evaluation of the subclavian arteries be desired. However, sagittal or oblique sagittal [left-anterior-oblique (LAO) view] prescriptions are recommended if higher resolution images are desired of the aortic arch as this will optimize anatomic coverage using the highest possible spatial resolution (ie, smallest voxel size). Sagittal acquisitions also have the advantage of facilitating the use of fractional or rectangular fields of view (ie, phase direction anterior to posterior) with minimal concerns from aliasing of the image data. A rectangular field of view shortens the overall scan time, thereby reducing the breath-holding time.

\section{Timing of Image Acquisition}

As mentioned, proper coordination of the central k-space views with peak enhancement of the target vessels is essential for Gd-enhanced 3D MRA. Imaging prematurely can result in incomplete vascular depiction. Imaging too late may result in insufficient signal within the target vessel.

Timing is more important for arterial imaging than venous imaging, as the arterial first pass of the bolus may be brief. Arterial phase Gd-enhanced 3D MRA can be timed several ways. The easiest method is using a fixed scan delay (ie, "best guess" or "educated guess") $(3,8,16)$. For a typical $30-40$ second sequentially ordered fast $3 \mathrm{D}$ gradient-echo pulse sequence with a 0.2 $\mathrm{mmol} / \mathrm{kg}$ contrast bolus (ie, 30-40 mL) injected at 2 $\mathrm{mL} / \mathrm{sec}$, the imaging of the thoracic aorta should begin approximately 10 seconds after the beginning of the intravenous infusion. For the pulmonary arteries, imaging should begin within 2-3 seconds after the contrast media injection has begun. Because timing mismatch is more often encountered with fixed time delay acquisitions, longer and larger contrast media infusions (ie, 0.2

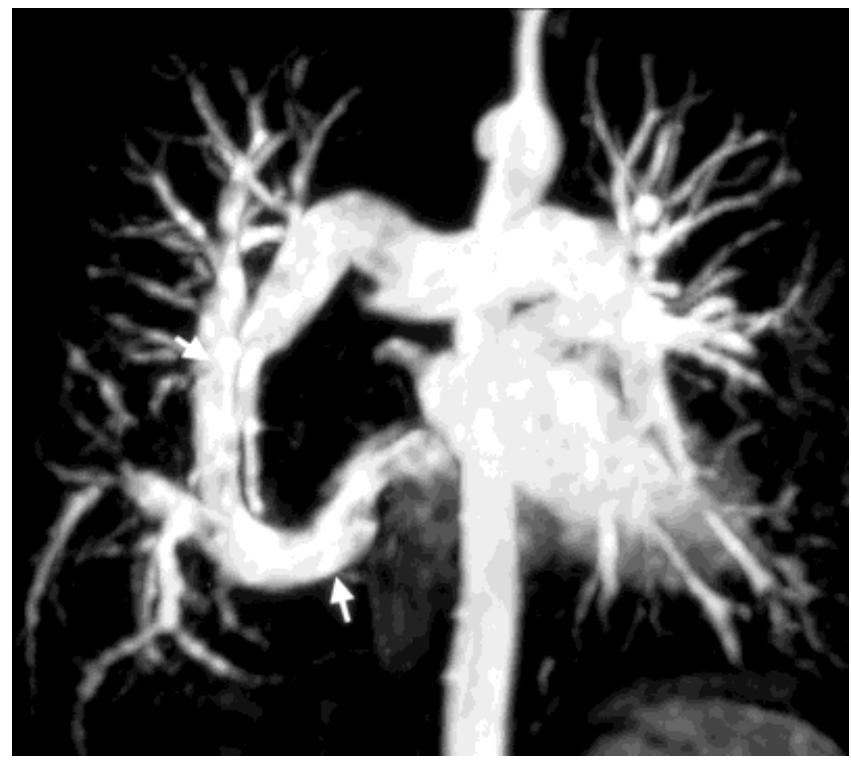

Figure 5. MIP from 3D Gd-MRA demonstrating large anomalous pulmonary vein (arrows) draining into the right atrium. $\mathrm{mmol} / \mathrm{kg}$ dose of contrast media) are recommended for this method.

A more "precise" method for estimating the arrival time of contrast media into the target vasculature is the performance of bolus timing scan using a 1-2 mL test bolus of contrast media $(16,19,25,26)$. Imaging is typically performed using a fast $2 \mathrm{D}$ gradient-echo pulse sequence over the target structures with a temporal resolution of approximately one image every 1-2 seconds. A large flip angle and either a preparatory pulse or image in the plane of the aorta are used to eliminate bright in-flow signal from the TOF effect. This preliminary scan will yield the time required for peak enhancement of the target vasculature. Using this information, the appropriate time delay to align the central $\mathrm{k}$-space lines with arterial enhancement can be calculated (8).

The third method for timing Gd-enhanced 3D MRA relies on the triggering of the image acquisition in real time. One such method (MR SmartPrep, General Electric Medical Systems, Milwaukee, WI) (27-29) monitors signal intensity within an operator-defined monitoring volume encompassing part of the target vasculature (eg, descending aorta for imaging the thoracic aorta) to determine when to initiate data acquisition. The monitoring volume can be placed in a cardiac chamber should pulmonary imaging be desired (29). The bolus detection algorithm has the advantage of automation. It also can be performed successfully with hand infusions. A similar approach using visual cues with MR fluoroscopy (30) has also been successfully implemented.

Fixed delay times are typically successful for Gdenhanced 3D MRA of the thoracic arteries. Timing the acquisition with a test bolus or real-time triggering method, however, can diminish the dose of contrast media (eg, to as low as $20 \mathrm{~mL}$ ) required for achieving diagnostic images. Irrespective of timing choice, two post-contrast 3D MRA acquisitions should be performed. The initial 3D MRA is typically performed during a breath-hold for the arterial phase images and the second 3D MRA immediately after the first (ie, second breath-hold) for the venous or more appropriately termed equilibrium phase imaging. The use of this dual-phase imaging in the thorax will assist segmentation of the arterial and venous structures. Even if pulmonary venous imaging is desired, the performance of coronal dual-phase images of the pulmonary circulation is recommended. The early arterial phase images (ie, images of the pulmonary arteries) can assist in the identification of arterial and venous segments, which are typically similarly bright on equilibrium phase images. The same dual-phase regimen is recommended for arterial imaging. Equilibrium phase images often compliment arterial phase images, which may insufficiently illustrate arterial structures because of arterial artifacts that occur on the initial arterial phase (Figs. 3, 6) or slow arterial flow (Fig. 7c,d). Dual-phase imaging often compensates for failures of timing and is a quick and inexpensive option.

To improve timing further, several investigators have proposed alternative methods of $\mathrm{k}$-space phase ordering. One strategy is to acquire the central lines of 

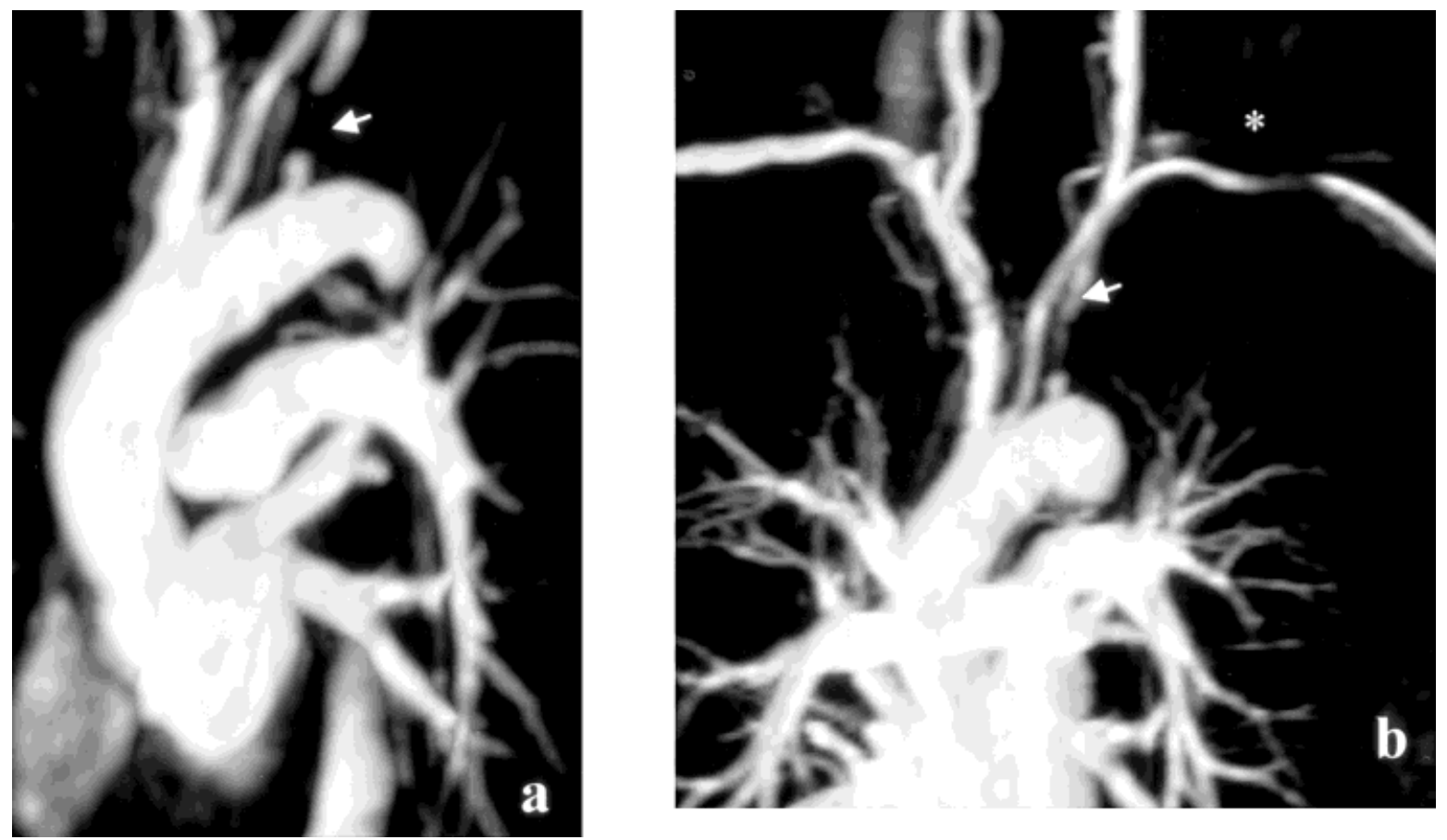

Figure 6. MIP reformats from coronal 3D Gd-MRA demonstrate severe stenosis of proximal left subclavian artery (arrow). a: Oblique sagittal plane. b: Coronal plane. Signal drop-off from concentrated gadolinium at mid-subclavian artery mimics stenosis (asterisk).

$\mathrm{k}$-space early during the imaging period $(27,30)$. This technique, called centric phase ordering, has theoretical advantages over traditional sequential phase ordering where the center $\mathrm{k}$-space views are acquired in the middle of the imaging period. Centric phase ordering enables the acquisition of the crucial image contrast data first. This can improve the ability to "catch" the arterial phase of arteries during brief periods of preferential enhancement (eg, carotid arteries) especially when implemented as elliptical centric $(19,20)$. This also may be beneficial for imaging patients with limited breathholding ability, ensuring that the critical central k-space data is acquired earlier during the breath-hold $(21,22)$. Incomplete breath-holding would thus only corrupt peripheral lines of $\mathrm{k}$-space and result in less significant degradation of image contrast.

Centric phase ordering of the 3D MRA, however, has disadvantages related to its sensitivity to fluctuations in signal intensity as seen during the arrival of Gd-chelate contrast media. Premature acquisition of images using a centric phase ordered Gd-enhanced 3D MRA can have significant ringing artifact. This artifact is recognized by alternating bright and dark bands, which parallel vascular structures and can render the study non-diagnostic. Alternative k-space methods such as elliptical centric phase ordering have been suggested to reduce these "ringing" artifact (21).

\section{Contrast Bolus}

Gd-chelate contrast media is typically administered intravenously via hand bolus or MR-compatible injector. In either case, the injection should be followed by a saline flush (typically 15-20 $\mathrm{mL}$ ) to ensure that the entire contrast media bolus has been administered. The right arm is the preferred intravenous site because it has a more direct path to the heart compared to the left arm (31). Kopka et al (32) have shown that an injection rate of $2 \mathrm{~mL} / \mathrm{sec}$ is ideal for Gd-enhanced 3D MRA. A minimum dose of at least $0.12 \mathrm{mmol} / \mathrm{kg}$ (8) (roughly 20 $\mathrm{mL}$ of Gd-chelate contrast media) is required for imaging the thoracic aorta, great vessels and pulmonary vasculature. Better quality is possible with higher doses, and we routinely use $40 \mathrm{~mL}$ for thoracic MRA.

Particular attention should be taken when evaluating the great vessels on Gd-enhanced 3D MRA. The arrival of contrast media in the subclavian and bracheocehpahlic veins is typically very concentrated and can transiently decrease T2*. This susceptibility or T2* artifact can dephase signal within the veins and adjacent structures (Figs. 3c, 6b). The arm contralateral to the suspected abnormal vessel should therefore be injected in order to minimize this artifact in the region of interest. In addition, use of a short echo time will also reduce the artifact. This artifactual signal drop-out is seen only during the arterial phase of the 3D Gd-MRA when the concentration of Gd is greatest. Inspection of the equilibrium phase images will help avoid erroneous diagnoses resulting from this artifact (Fig. 3d). When it is important to see subclavian arteries, it may be useful to use a fast injection of a lower dose $(20 \mathrm{ml})$ followed immediately by a fast saline flush ( $50 \mathrm{ml}$ at $5 \mathrm{ml} / \mathrm{sec}$ ) to clear gadolinium from the subclavian vein before central $\mathrm{k}$-space data are acquired. If venous visualization is the target of the study, a high dose of contrast media (eg, 40 $\mathrm{mL}$ ) should be administered for Gd-enhanced 3D MRA 

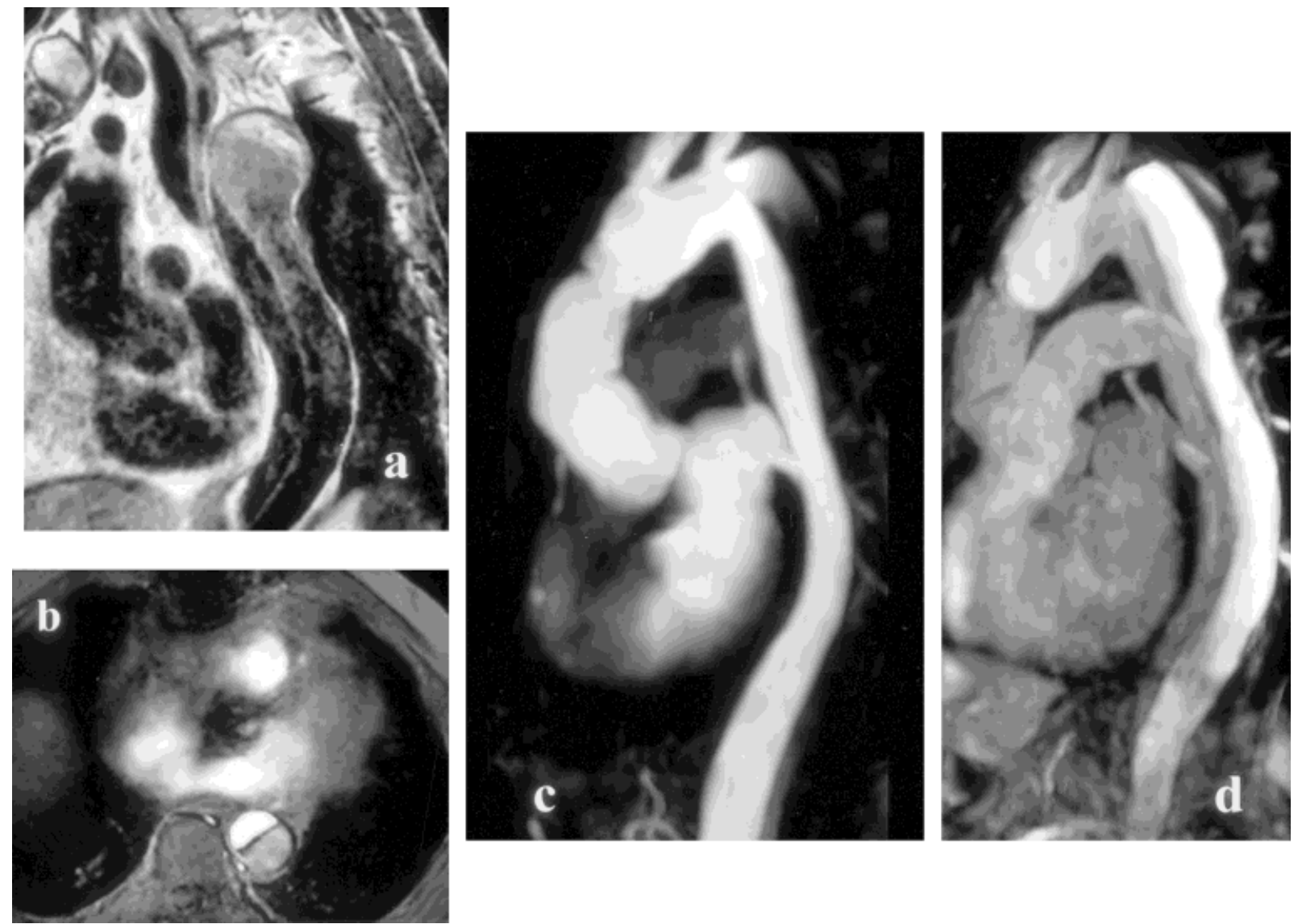

Figure 7. Aortic dissection type III (Stanford B). The intimal flap demonstrated as linear intermediate signal in the sagittal spin-echo sequence (a), and as low signal in axial 2D TOF (b). The slow flow in the false lumen has lower signal than the normal flow in the true lumen (b). Early filling of the true lumen in arterial phase (c). Compared with delayed filling of the false lumen in the equilibrium phase (d).

to ensure sufficiently high venous concentrations of $\mathrm{Gd}$ during the equilibrium phase imaging.

An alternative method for central venous imaging is to administer a diluted dose of Gd-chelate contrast media directly into the vein of interest. For this alternate venographic technique, $10-20 \mathrm{~mL}$ of Gd-chelate contrast media is mixed into a $250 \mathrm{~mL}$ bag of normal saline. A $120 \mathrm{~mL}$ bolus (requires two $60 \mathrm{ml}$ syringes) of the diluted Gd-chelate contrast media should be administered intravenously into an antecubital vein of the symptomatic extremity at a rate of at least $2 \mathrm{~mL} / \mathrm{sec}$. Imaging should start within 5-10 seconds after the initiation of the contrast injection, with the patient suspending breathing in full inspiration. For bilateral upper extremity illustration, two $120 \mathrm{~mL}$ injections of the diluted contrast media (ie, total of $240 \mathrm{~mL}$ ) should be injected simultaneously (120 mL into each arm).

\section{Gd-Enhanced 3D MRA Image Processing}

Gd-enhanced 3D MRA provides high-resolution images of the vessels as "luminograms" and can be displayed as maximum intensity projections (MIP), which are views similar to those of conventional angiography-views familiar to clinicians. A major advantage of Gd-enhanced 3D MRA over conventional angiography, which is limited to planar $2 \mathrm{D}$ image display, is the ability of
Gd-enhanced 3D MRA data to be reformatted into planes different from the original acquisition. MPR and subvolume MIPs are a particularly helpful tool for the evaluation of the thoracic vessels, which are often overlapping.

\section{Patient Preparation}

Preparing the patient for a Gd-enhanced 3D MRA examination is an important step. As always, the patient's fears should be allayed prior to imaging, and communication with the patient to include an explanation of the procedure (especially of the breath-holding) should be initiated prior to scanning. A preliminary breath-hold prior to imaging can serve as a guide to the patient's breath-hold capacity. Using supplemental oxygen and hyperventilation, most patients, even those with respiratory disease, can hold their breath for 20-30 seconds (34). Reasonably healthy individuals can suspend breathing 40-50 seconds without difficulty when lying down relaxed inside the magnet.

The placement of a 20 gauge intravenous angiocatheter prior to entering the scan room will minimize the scanner time requirements. Similarly, the setup of the intravenous tubing set and drawing of the saline flush and contrast media in advance will also diminish the demands on scanner time. It is helpful to use a standard- 
ized tubing setup so the operator will know the tubing dead space and the flow resistance to contrast injection.

The patient is typically positioned feet first with head toward the operator. This allows more direct communication between the operator and the patient. Positioning of the patient feet first also facilitates the operator's visualization of the patient's anterior chest wall and respiratory excursion to verify that the patient is following breathing instructions. Placement of a respiratory bellows around the patient's chest can be helpful for monitoring the patient's respiratory cycle and estimating potential breath-hold capacity. However, the delay in scanner display of the respiratory bellows data is typically several seconds, and direct observation of the patient's breathing is often a more accurate method for the initiation of breath-hold scanning.

Imaging can be improved by the use of a torso phasedarray coil. However, the phased-array coil will restrict the cranial-to-caudal extent of the imaging field of view. Larger fields of view may be necessary for imaging a patient with a thororacoabdominal aortic aneurysm or a thoracic aortic dissection, which extends into the abdominal aorta. Use of the phased-array coils often adds to setup time, as additional care must be taken to ensure that it is properly centered prior to the Gdenhanced 3D MRA. Another consideration of phased- array coils is the time required for the processing of the image data, which can be significant if the scanner does not have sufficiently fast array processors or memory. In most cases, the body coil is adequate for thoracic Gd-enhanced 3D MRA and is the simplest coil choice.

\section{THORACIC APPLICATIONS OF Gd-ENHANCED 3D MRA}

Gd-enhanced 3D MRA is a versatile technique for imaging thoracic arteries and veins. In the sections to follow, potential applications for Gd-enhanced 3D MRA of the systemic circulation (thoracic aorta and central veins) and pulmonary circulation (pulmonary arteries and pulmonary veins) will be briefly discussed and illustrated. In addition, MRA for the delineation of aberrant systemic-to-pulmonary circulation connections will also be depicted.

\section{Systemic Circulation}

Thoracic Aorta

One of the most common vascular indications for thoracic MRI is the evaluation of known or suspected aortic pathology. Although a variety of entities can affect the aorta, imaging should not only identify the lesions but
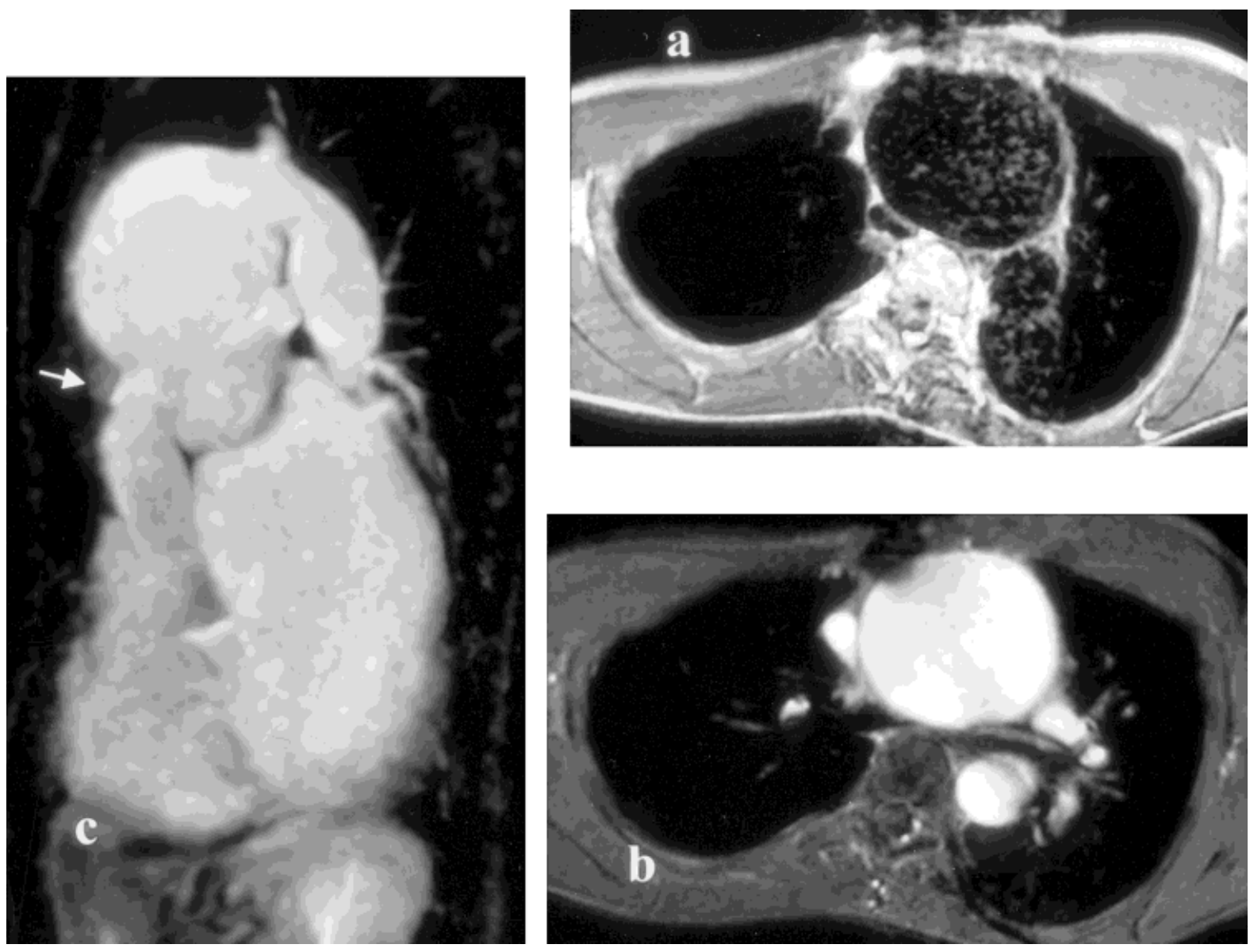

Figure 8. Aneurysm of the ascending aorta in Marfan' syndrome. a: Axial T1 SE. b: Axial 2D TOF. c: Sagittal MIP projection. The arrow points to the site of anastomosis between the native aneurysmal aorta and the graft placed in prior surgery to correct the aortic root aneurysm (c). Note associated chest wall deformity $(\mathrm{a}, \mathrm{b})$. 
also accurately characterize their location, extent, and involvement of branch vessels. In addition, the examination must be able to assess the salient features required for surgical planning.

MRI of an aortic aneurysm (Fig. 8) should demonstrate the site of aneurysm, its length, morphology (ie, fusiform versus saccular), and relationship to aortic branches. From these features, the etiology can often be determined. Atherosclerotic aneurysms, for example, can occur anywhere, but typically involve the descending aorta and are fusiform. Mycotic aneurysms (Fig. 9) are commonly saccular and involve the ascending aorta, arch or the aorta near the diaphragm. In addition, images should illustrate the relationship of the aneurysm to the branch vessels and the presence of mural thrombus or a penetrating ulcer, all of which can affect surgical decision making. T1-weighted spin-echo and gradient-echo images can often be sufficient for the simple identification of a suspected aneurysm (ie, is there an aortic aneurysm?) or follow-up assessment of known aneurysms to monitor its size. However, Gdenhanced 3D MRA provides more comprehensive information more reliably and faster than these other methods without even requiring electrocardiographic gating.

Similar observations can be made for MRI of aortic dissections. In fact, the location and relationship of the intimal tear is critical to the choice of management. Aortic dissections involving the ascending aorta (Stan- ford A, Fig. 10a) are associated with higher mortality and mandate surgical repair. Stanford A dissections have increased risk for myocardial infarct secondary to extension of the dissection into the coronary arteries, of pericardial temponade secondary to aortic rupture into the pericardium, and possibly of stroke secondary to involvement of the carotid or vertebral arteries. Dissections arising distal to the left subclavian (Stanford B, Figs. 7, 10b) are less precarious and are usually managed medically. MRA must evaluate the extent of dissection, entry and reentry sites, true and false channel size and patency, and branch vessel involvement. Because of the possibilities for an aortic dissection without intimal flap (intramural hematoma) where a discrete intimal tear is not evident, pre-contrast $\mathrm{T} 1$-weighted spin-echo imaging is necessary. On T1-weighted spinecho images, the intramural hematoma is identified as concentric thickening of the aortic wall with increased intramural signal intensity, which is related to methemoglobin within the hematoma (35).

Stenosis or occlusion of the great vessels may be caused by atherosclerosis, dissection, and arteritis (Takayasu's arteritis, giant cell arteritis). Other rare causes include fibromuscular dysplasia, post-radiation arteritis, and mediastinal inflammatory or neoplastic disease. The patient commonly presents with upper extremity ischemia and/or neurologic symptoms from the steal phenomena during arm exercise. Coronal 3D
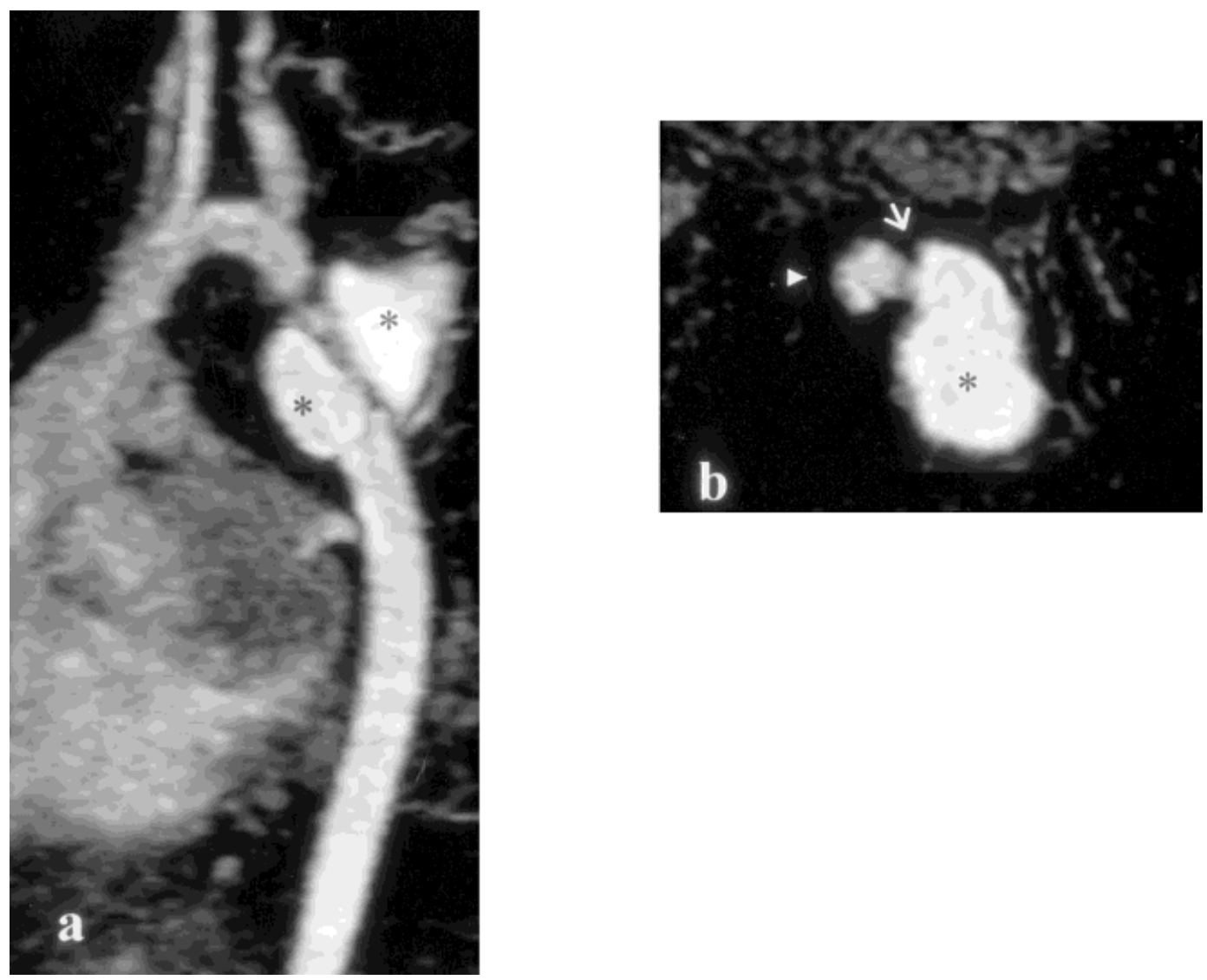

Figure 9. MIP reformats from coronal 3D Gd-MRA demonstrate a mycotic pseudoaneurysm at the site of coarctation, ruptured as a complication from bacterial endocarditis in (a) sagittal and (b) axial planes. Pseudoaneurysm lumen (asterisk), true lumen (arrowhead), and the neck (arrow). 

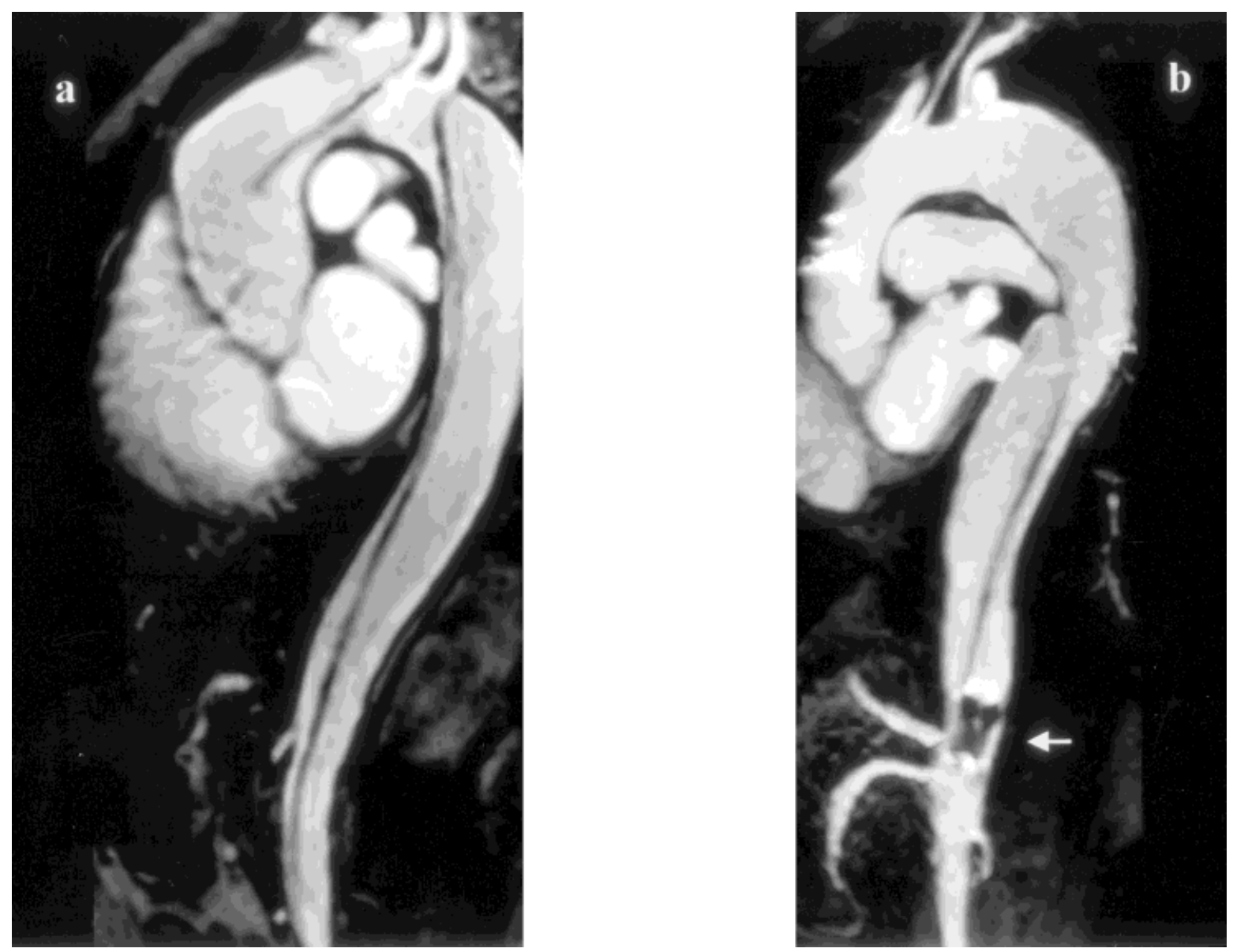

Figure 10. MIP reformats from coronal 3D Gd-MRA show aortic dissections with intimal flap. a: DeBakey type 1 (Stanford type A) dissection. b: DeBakey type 3 (Stanford type B) dissection. Note susceptibility artifact from the metallic stent (arrow).
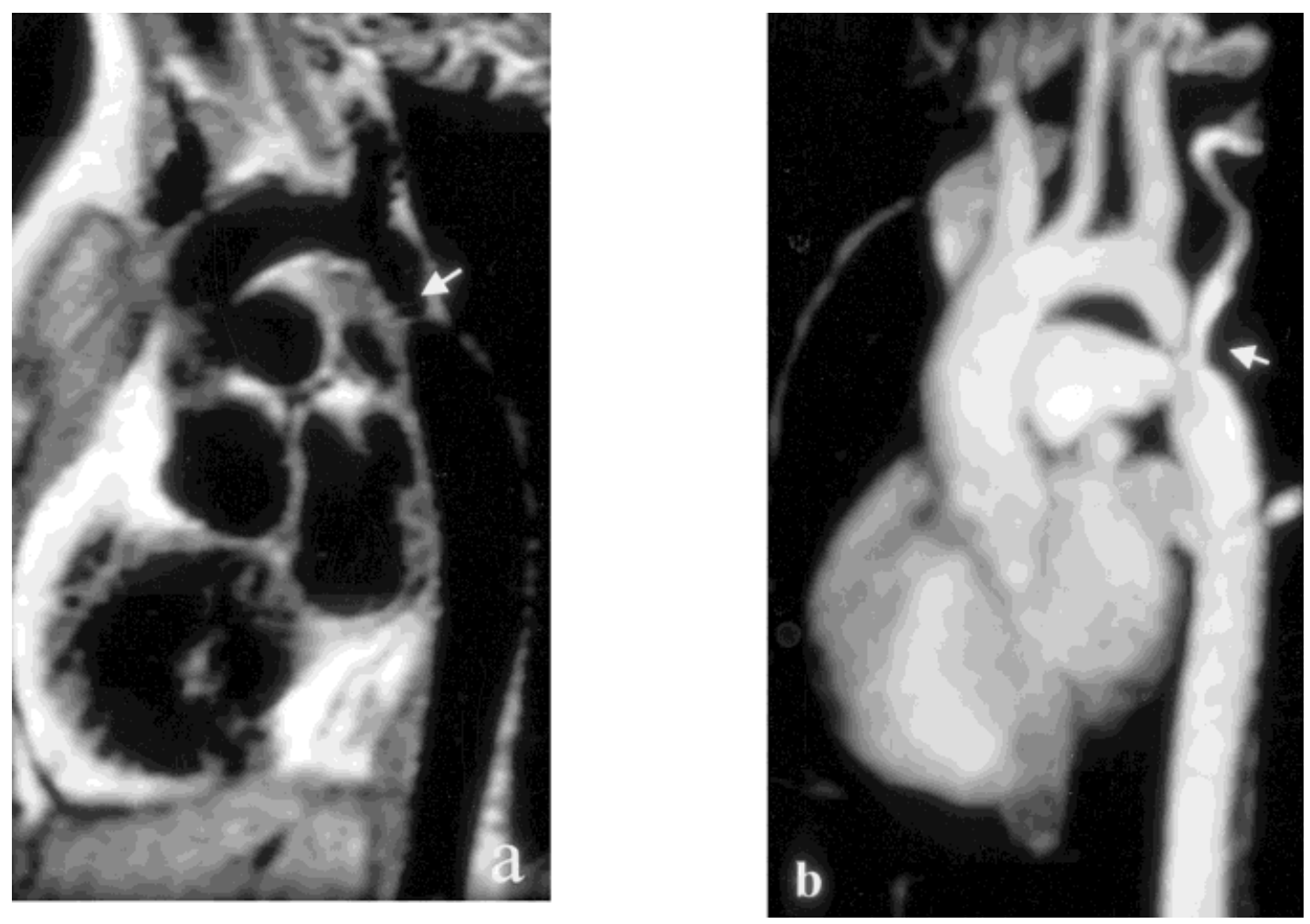

Figure 11. Sagittal spin echo (a) and 3D Gd-MRA reformats (b) of the thoracic aorta demonstrate aortic coarctation (arrows) distal to the left subclavian artery origin. Note the large collateral bypassing the coarctation. 
Gd-MRA is the best sequence to assess origins of the innominant artery, common carotids, vertebral, and subclavian arteries (Fig. 6). The shortest possible echo time should be used to minimize the susceptibility artifact from the lung and from the concentrated gadolinium in the subclavian and/or innominate veins. Inject the gadolinium via the arm contralateral to the suspected abnormal vessel to avoid artifact from highly concentrated gadolinium in the veins which can mimic stenosis in the subclavian and/or innominate arteries.

Less commonly, patients with suspected congenital lesions such as vascular rings or aortic coarctation are referred for MRI. Standard T1-weighted spin-echo and gradient-echo images frequently are sufficient for confirming a vascular ring (Fig. 2) or aortic narrowing. In the case of aortic coarctation, Gd-enhanced 3D MRA can provide important complimentary information (Fig. 11). Signal drop in the aorta distal to the coarctation may be seen on cine MRA (gradient echo) indicating that the stenosis is significant. This signal drop is caused by flow turbulence distal to the stenosis, resulting in spin dephasing. The degree of dephasing provides a rough qualitative assessment of the severity of stenosis and helps in differentiating a pseudocoarctation (Fig. 12) from a true coarctation of the aorta. Quantitative measurements can be obtained by using velocity-encoded cine MRA, which allows pressure gradient measures across the coarctation. In addition, velocity-encoded

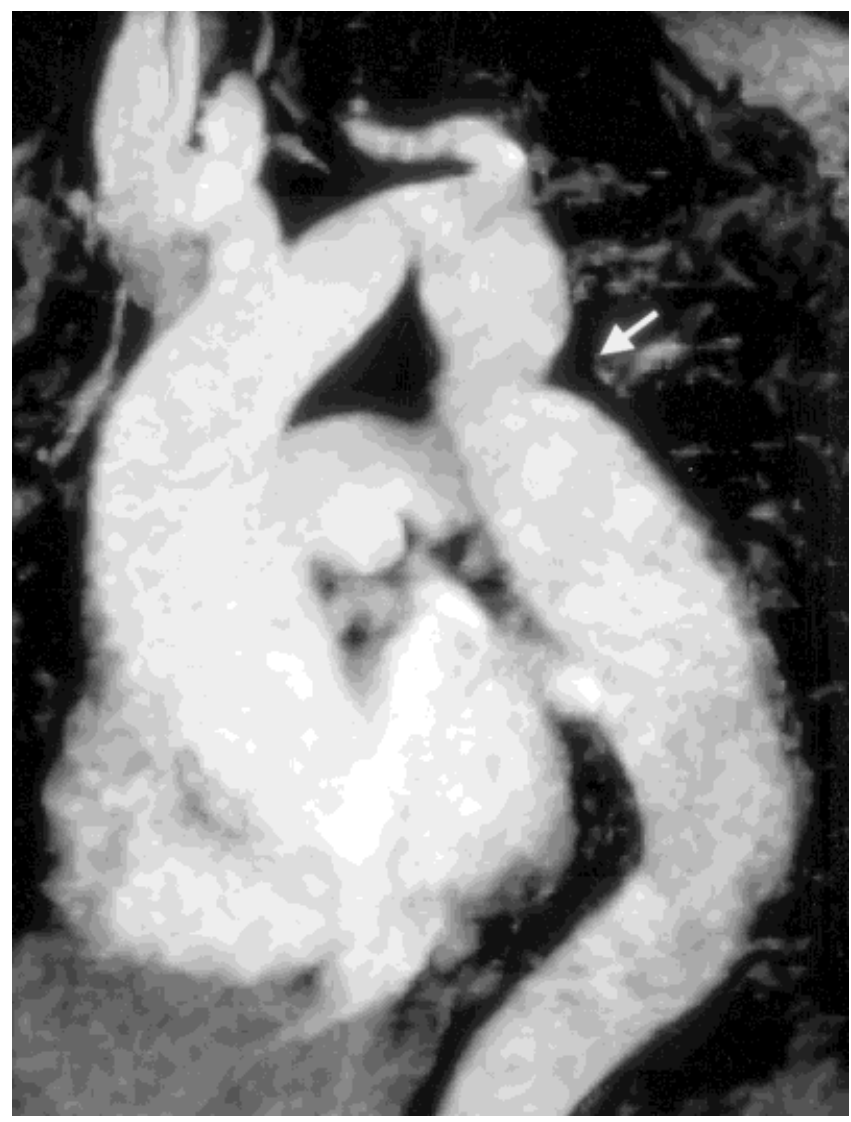

Figure 12. Pseudocoarctation of the thoracic aorta (arrow) on sagittal oblique subvolume MIP. Note the high aortic arch and unusually large A-P window typical of pseudocoarctation in which the aorta becomes ectatic and elongated with kinks that resemble coarctation but are not hemodynamically significant. Also note that no collaterals are present. cine MRA can demonstrate the reversed flow pattern in the descending aorta distal to the coarctation, due to collateral flow (28).

Other congenital anomalies of the aorta including aortopulmonary window, aberrant subclavian arteries, patent ductus arteriosus, and interrupted arch are also well identified on MRA $(2,29)$.

\section{Bracheocephalic Veins and Superior Vena Cava}

MRI of the central veins is typically performed for the assessment of venous patency, for which traditional spin-echo and gradient-echo (especially TOF technique) images are often sufficient (Fig. 1) (36). Venous imaging can be achieved during the equilibrium phase of a coronal Gd-enhanced 3D MRA using a higher dose of contrast media or during the initial (arterial) phase of a coronal Gd-enhanced 3D MRA using a injection of diluted contrast media bolus into the ipsilateral arm.

\section{Pulmonary Circulation}

\section{Pulmonary Arteries}

A common clinical indication for pulmonary artery imaging is the evaluation of patients suspected of having a pulmonary embolism. The use of traditional MRI pulse sequences for imaging the pulmonary vessels presents a great challenge due to the large local dephasing resulting from multiple air-soft tissue interfaces, as well as artifacts related to cardiac and respiratory motion $(23,37)$. The main pulmonary artery and the right and left pulmonary arteries can usually be seen on T1-weighted spin-echo sequences with the assistance of respiratory and cardiac gating. Thrombus appears as intermediate signal within the signal void pulmonary artery. However, the varying flow direction within these vessels as well as the potentially diminished flow may lead to incomplete wash-out and to the erroneous simulation of a thrombus.

More recently, Gd-enhanced 3D MRA has been demonstrated for imaging patients with suspected pulmonary embolism (Fig. 13). Gd-enhanced 3D MRA is especially helpful for imaging patients with indeterminate pulmonary ventilation and perfusion (ie, V/Q) scintigraphic studies and contraindications to the use of iodinated contrast agents. Several studies have reported high sensitivity and specificity for Gd-enhanced 3D MRA in detecting pulmonary embolism up to the subsegmental branches $(22,23,38,39)$.

\section{Pulmonary Veins}

MRI of the pulmonary veins is a fairly infrequent request but has been shown to be helpful for the evaluation of anomalous pulmonary venous return in patients with suspected congenital heart disease (30). The pulmonary veins, which carry oxygenated blood, normally drain into the left atrium, where blood is pumped into the left ventricle and the systemic arterial circulation. The pre-operative determination of pulmonary venous drainage is important for certain surgical repairs such as atrial septal defects, which have a higher association with anomalous pulmonary venous drainage. Standard T1-weighted spin-echo and gradient-echo images often 

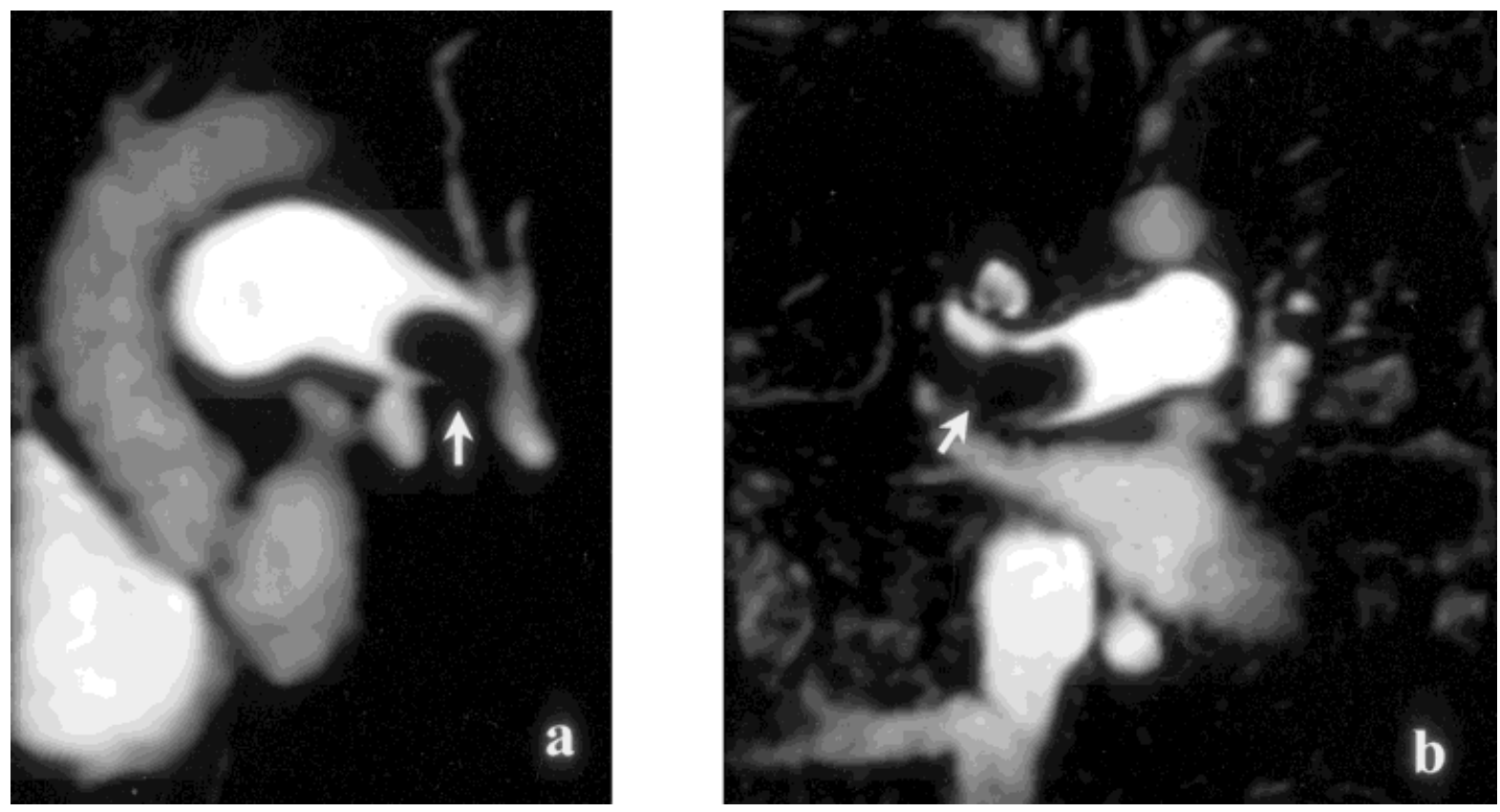

Figure 13. MIP reformats from coronal 3D Gd-MRA show a non-occlusive thrombus (arrow) of the left main stem pulmonary artery in (a) sagittal and (b) coronal planes.

have insufficient contrast-to-noise ratio and spatial resolution to differentiate the individual lobar pulmonary veins accurately from their accompanying arteries. This is especially true in children, for whom most of these studies are requested. Gd-enhanced 3D MRA can provide excellent depiction of the pulmonary veins (Fig. 5) and compares favorably with and is arguably better than images obtained during the venous phase (ie,
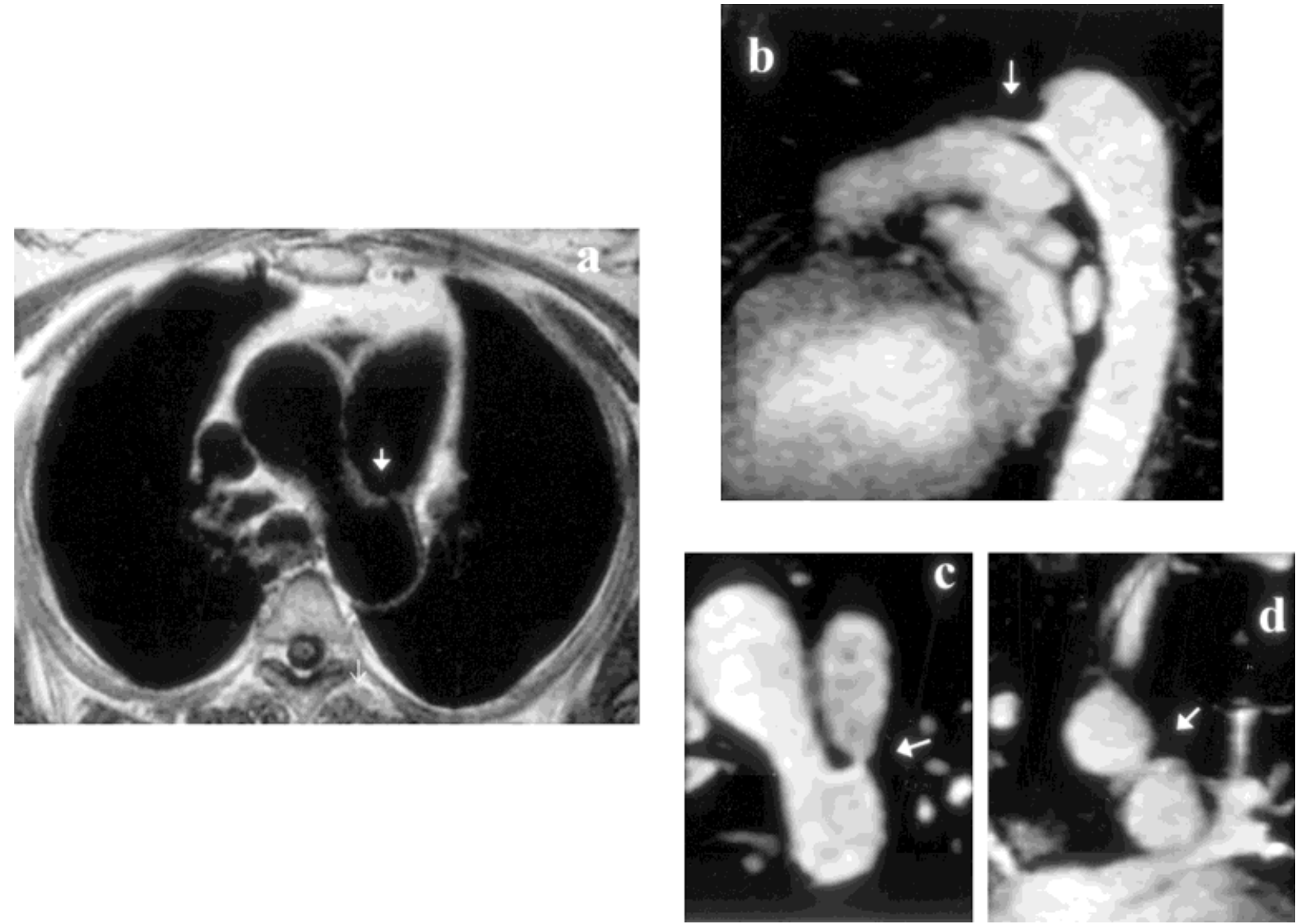

Figure 14. Patent ductus arteriosus in (a) axial spin echo and (b-d) MIP from coronal 3D Gd-MRA demonstrates the communication between the aorta and main pulmonary artery (arrow). 
levo-phase) of a conventional pulmonary angiogram (41).

\section{Systemic-Pulmonary Circulation Connections}

With the advantages of high-resolution 3D data and MPR, Gd-enhanced 3D MRA can often define smallcaliber vessels or vascular channels not well visualized on T1-weighted or gradient-echo images. It is not surprising, therefore, that this technique can be particularly helpful for the identification of systemic-pulmonary circulation connections (ie, shunt lesions), which may often be subtle. Intracardiac shunts (eg, atrial septal defects and ventricular septal defects) are usually well delineated using T1-weighted spin-echo and gradient-echo images. However, these flow-based sequences often fail to delineate extracardiac shunts adequately because they supply insufficient contrast-tonoise ratio or the flow within the shunts is too complex for them to generate sufficient signal. Gd-enhanced 3D MRA can often better define these anomalous connections (Fig. 14) because they generally will fill with contrast media. MPR untangles overlapping structures for better visualization of the anomalous connection. Gd-enhanced 3D MRA also has the advantages of MIP display for presentation of views familiar to the surgeon.

\section{REFERENCES}

1. Amparo EG, Higgins CB, Hricak H, Sollitto R. Aortic dissection: Magnetic resonance imaging. Radiology 1985;155:399-406.

2. Geisinger MA, Risius B, O'Donnell JA, et al. Thoracic aortic dissection: Magnetic resonance imaging. Radiology 1985:407-412.

3. Glazier HS, Gutierrez FR, Levitt G, Lee JKT, Murphy WA. The thoracic aorta studied by MR imaging. Radiology 1985;157:149155.

4. Nayler GL, Firmin DN, Longmore DB. Blood flow imaging by cine magnetic resonance. J Comput Assit Tomogr 1986;715-722.

5. Bisset III GS. Pediatric thoracic applications for magnetic resonance imaging. J Thorac Imaging 1989;4:51-57.

6. Link KM, Lesko NM. The role of MR imaging in the evaluation of acquired diseases of the thoracic aorta. AJR 1992;158:1115-1125.

7. Nienaber CA, von Kodolitsch Y, Nicholas V, et al. The diagnosis of thoracic aortic dissection by noninvasive imaging procedures. $\mathrm{N}$ Engl J Med 1993;328:1-9.

8. Didier D, Ratib O, Friedli B, et al. Cine gradient-echo MR imaging in the evaluation of cardiovascular diseases. RadioGraphics 1993;13: 561-573.

9. Hartnell GG, Finn JP, Zenni M, et al. MR imaging of the thoracic aorta: Comparison of spin-echo, angiographic, and breath-hold techniques. Radiology 1994;191:697-704.

10. Higgins CB. Thoracic Aortic Disease. In: Higgins CB (ed). Essentials of cardiac radiology and imaging. Philadelphia: JB Lippincott Company; 1992;179-214.

11. Listerud J. First principles of magnetic resonance angiography. Magn Reson Q 1991;7:136-170.

12. Atkinson D, Teresi L. Magnetic resonance angiography. Magn Reson $\mathrm{Q}$ 1994;10:149-172.

13. Prince MR, Yucel EK, Kaufman JA, Harrison DC, Geller SC. Dynamic gadolinium-enhance three-dimensional abdominal MR arteriography. J Magn Reson Imaging 1993;3:877-881.

14. Prince MR. Gadolinium-enhanced MR aortography. Radiology 1994; 191:155-164.

15. Maki JH, Prince MR, Londy FJ, Chenevert TL. The effect of time varying intravascular signal intensity and $\mathrm{k}$-space acquisition order on three dimensional MR angiography image quality. J Magn Reson Imaging 1996;6:642-651.

16. Hany TF, Mckinnon GC, Leung DA, Pfannatter T, Debatin JF. Optimization of contrast timing for breath-hold three dimensional MR angiography. J Magn Reson Imaging 1997;7:551-556.

17. Prince MR. Contrast-enhanced MR angiography: Theory and optimization. MRI Clin North Am 1998;6:257-267.
18. Prince MR, Narasimham DL, Jacoby WT, Williams DM, Cho KJ, Marx MV, Deeb GM. Three-dimensional gadolinium-enhanced MR angiography of the thoracic of the thoracic aorta. AJR 1996;166: 1387-1397.

19. Krinsky G, Rofsky N, Flyer M, et al. Gadolinium-enhanced threedimensional MR angiography of acquired arch vessel disease. AJR 1996; 167:981-987.

20. Krinsky GA, Rofsky N, Flyer M, et al. Thoracic aorta: Comparison of gadolinium-enhanced three-dimensional MR angiography with conventional MR imaging. Radiology 1997;202:183-193.

21. Ho VB, Prince MR. Thoracic MR aortography: Imaging technique and strategies. RadoGraphics 1998;18:287-309.

22. Meaney JFM, Weg JG, Chenevert TL, Stafford-Johnson D, Hamilton $\mathrm{BH}$, Prince MR. Diagnosis of pulmonary embolism with magnetic resonance angiography. N Engl J Med 1997;336:1422-1427.

23. Kauczor HU. Contrast-enhanced magnetic resonance angiography of the pulmonary vasculature. Invest Radiol 1998;33:606-617.

24. Du YP, Parker DL, Davis WL, Cao G. Reduction of partial-volume artifacts with zero-filled interpolation in three-dimensional MR angiography. J Magn Reson Imaging 1994;4:733-737.

25. Earls JP, Rofsky NM, DeCorato DR, Krinsky GA, Weinreb JC Breath-hold single-dose gadolinium-enhanced three-dimensional MR aortography: Usefulness of a timing examination and MR power injector. Radiology 1996;201:705-710.

26. Kim JK, Farb RI, Wright GA. Test bolus examination in the carotid artery at dynamic gadolinium-enhanced MR angiography. Radiology 1998;206:283-289.

27. Prince MR, Chenevert TL, Foo TKF, Londy FJ, Ward JS, Maki JH. Contrast-enhanced abdominal MR angiography: Optimization of imaging delay time by automating the detection of contrast material arrival in the aorta. Radiology 1997;203:109-114.

28. Foo TKF, Saranathan M, Prince MR, Chenevert TL. Automated detection of bolus arrival and initiation of data acquisition in fast, three-dimensional, gadolinium-enhanced MR angiography. Radiology 1997;203:275-280.

29. Ho VB, Foo TKF. Optimization of gadolinium-enhanced MRA utilizing an automated bolus detection algorithm (MR SMARTPREP). Invest Radiol 1998;33:515-523.

30. Wilman AH, Riederer SJ, King BF, Debbins JP, Rossman PJ, Ehman RL. Flouroscopically triggered contrast-enhanced three-dimensional MR angiography with elliptical centric view order: applicaton to the renal arteries. Radiology 1997;205:137-146.

31. Lee YJ, Chung TS, Joo JY, Chien D, Laub G. Suboptimal contrastenhanced carotid MR angiography from the left brachiocephalic venous stasis. J Magn Reson Imaging 1999; 10:503-509.

32. Kopka L, Vosshenrich R, Rodenwaldt J, Grabbe E. Differences in kinjection rates on contrast-enhanced breath-hold three dimensional MR angiography. AJR 1998;170:345-348.

33. Hany TF, Schmidt M, Hilfiker PR, et al. Optimization of contrast dosage for gadolinium-enhanced 3D MRA of the pulmonary and renal arteries. J Magn Reson Imaging 1998;16:901-906.

34. Marks B, Mitchell DG, Simelaro JP. Breath-holding in healthy and pulmonary-compromised populations: effects of hyperventilation and oxygen inspiration. J Magn Reson Imaging 1997;7:595-597.

35. Murray JG, Manisali M, Flamm SD, et al. Intramural hematoma of the thoracic aorta: MR image findings and their prognostic applications. Radiology 1997;204:349-355.

36. Hartnell GG, Hughes LA, Finn JP, Longmaid III HE. Magnetic resonance angiography of the central chest veins: A new standard? Chest 1995; 107:1053-1057.

37. Erdman WA, Peshock RM, Redman HC, et al. Pulmonary embolism: Comparison of MR images with radionuclide and angiographic studies. Radiology 1994;190:499-508.

38. Gupta A, Frazer CK, Ferguson JM, et al. Acute pulmonary embolism diagnosis with MR angiography. Radiology 1999;210:353-359.

39. Hatabu H. MR pulmonary angiography and perfusion imaging: recent advances. Semin Ultrasound CT MR 1998;18:349-361.

40. Ho VB, Kinney JB, Sahn DJ. Contributions of Newer MR Imaging Strategies for Congenital Heart Disease. RadioGraphics 1996;16: 43-60.

41. Eisenhauer MD, Kinney JB, Ho VB, Braxton M, Peake JB. Preoperative gadolinium-enhanced magnetic resonance pulmonary venography in an adolescent with ostium secundum atrial septal defect. Military Medicine 1997;162:640-642. 